\title{
A METHODOLOGY TO CONDUCT A SYSTEMATIC REVIEW OF VIETNAMESE STUDIES ON SUSTAINABILITY DURING THE 2008- 2020 PERIOD
}

\author{
Ngoc-Thang B. Le; Manh-Tung Ho \\ Ritsumeikan Asia Pacific University \\ Верри City, Oita Prefecture, Japan \\ October $3^{\text {rd }, 2021}$
}

\begin{abstract}
To ensure a transparent reporting of systematic review, we follow the PRISMA 2020 guideline for systematic review (Page et al., 2021). We first identified the theme of this systematic review with database, selected keywords, and inclusion criteria, then narrowed down the qualified publications through titles, abstracts, and full-text manuscript examination. The PRISMA 2020 statement consists of a checklist of 27 items to cover in a systematic review and a flow diagram to visualize the reviewing process.
\end{abstract}




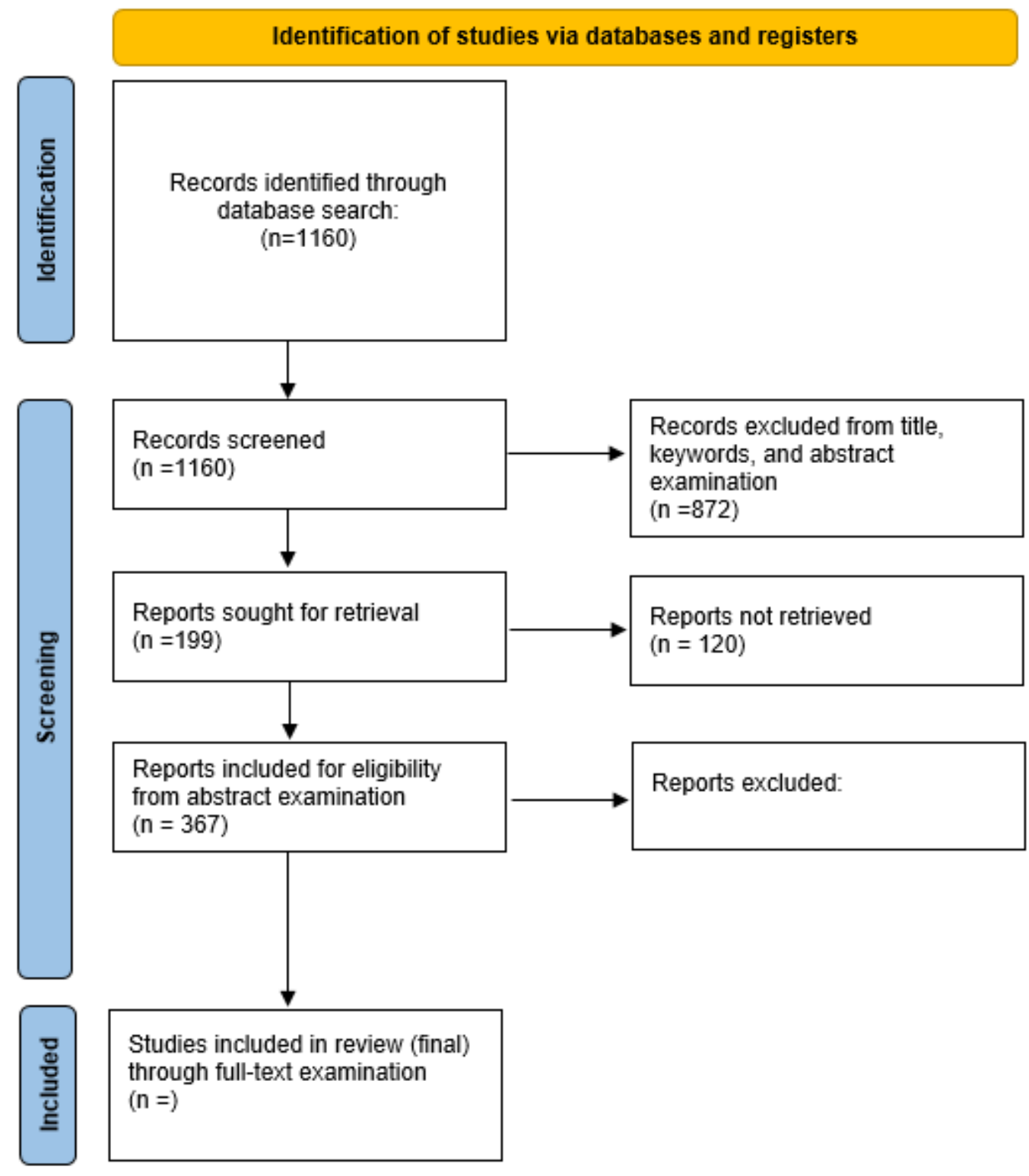

Fig 1. PRISMA flow diagram demonstrating the data collection and analysis process

\section{The SSHPA Database}

The database we use for this review is SSHPA (Social Sciences \& Humanities Peer Award), which is a nationally verified, structured, comprehensive open database specialized in social sciences and humanities research in Vietnam. This database has served to promote the 
transparency of science (Vuong, 2020; Teixeira da Silva and Vuong, 2021; Pham and Vuong, 2009) and reducing the cost of doing science (Vuong, 2018). Since 2008, all publications by Vietnamese scholars in the domains of social sciences and humanities that have appeared in Web-of-Science and Scopus-indexed academic journals have been tracked and documented in the database system. A more detailed description of a rigorous data processing procedure and the system's potential for reproducibility can be found in the data descriptor paper published in Nature Research's Scientific Data, a top-tiered data science journal (Vuong et al., 2018), or in the method article published in MethodsX. (Vuong et al., 2020). SDA is the short form of SSHPA Data Analysis. SDA extracts data from the SSHPA database and then generates a large array of visualizations based on this data. By implementing these two processes, frequent issues such as duplicate data, erroneous DOI, inaccurate author names, and so on will be eliminated, ensuring the correctness and precision of the results, which can then be used for various research objectives. The search strategy and data-filtering method will next be discussed.

\section{Search Strategy}

We decide to apply a systematic approach to our analysis for two main reasons. (1) The systematic approach allows us to examine a huge amount of data while focusing on empirical evidence and research output, which is crucial for determining the characteristics of the sustainable research database. (2) While the systematic approach is highly time-consuming, the features of our SSHPA database, such as the presentation of abstracts, keywords, and major outputs, will help to speed up the information-gathering process.

With the scope to understand the research landscape of sustainable living and its interconnection with climate change, we established a set of fundamental keywords that include "sustainability", "sustainable", "climate change", "environment". In the later brainstorming process for more definite keywords, we selected "natural disaster", “environmental crisis", “energy crisis", "green", "eco", "reduce”, "reuse", "recycle” on two bases: (1) to yield publications that emphasize the challenging aspects of climate change and (2) to yield publications that provide initiatives to the unsustainability issues. We entered the keywords in SSHPA's search bar individually, collected every matching publication, and extracted the dataset in the form of Excel CSV, which consists of 1160 publications. 


\section{Inclusion and exclusion criteria}

With SSHPA's precision of results and collection of English-based publications, the filtering process took place immediately after database extraction. We divided this process into two major procedures: (1) filter through titles and keywords, and (2) filter through extensive abstract examination. The qualified papers must satisfy two conditions: The study must be conducted in Vietnam or through secondary datasets recorded in Vietnam, and the study must contribute to the process of combating climate change and promoting sustainable living. In the first step, we retrieve ( ) papers from the dataset. A large number of the first filtering process should be reasonable as we wanted to archive as many potential papers as possible. The qualified papers would continue to go through an extensive abstract examination, where we checked abstracts for methodologies, results, discussions, and limitations of the studies if applied. Studies with omitted abstracts were automatically eliminated. We continued by categorizing the selected papers into their potential domains, identifying their themes and essential keywords. By the end of the second filtering process, the dataset consisted of 367 , and we identified the four pillars of this systematic review: Adaptation to climate change, Social sustainability, Financial Sustainability, and Education for Sustainable Development. Finally, we conducted extensive thematic reviews on each pillar through full-text manuscripts' examinations.

In the group of authors, three members took the main role of processing the database. To tackle the risk of authors' bias in selecting qualified publications, the processed datasets were crosschecked in-between authors and finalized by the first author. In addition, qualified studies must have clear effect measures in the result section and be valued to have significant findings and contributions to the understanding of their entitled pillar. As a result, studies with no direct results, lack of coherence between results and discussions, or lack of robustness in methodologies were manually eliminated. This criterion proved to be critical at the step of extensive thematic reviews, as we included only () papers with well-designed studies and significant results.

\section{References}


Page, M. J., Moher, D., Bossuyt, P. M., et al. (2021). PRISMA 2020 explanation and elaboration: updated guidance and exemplars for reporting systematic reviews. BMJ, 372, n160. https://doi.org/10.1136/bmj.n160

Phạm Minh Chính, Vương Quân Hoàng. (2009). Kinh tế Việt Nam: Thăng trầm và đột phá. Nxb Chính trị Quốc gia, Hà Nội.

Teixeira da Silva, J. A., Vuong, Q. H. (2021). The right to refuse unwanted citations: rethinking the culture of science around the citation. Scientometrics, 126, 5355-5360. https://doi.org/10.1007/s11192-021-03960-9

Vuong, Q. H. (2018). The (ir)rational consideration of the cost of science in transition economies. Nature Human Behaviour, 2, 5. https://doi.org/10.1038/s41562-017-0281-4

Vuong, Q. H. (2020). Reform retractions to make them more transparent. Nature, 582, 149. https://doi.org/10.1038/d41586-020-01694-X

Vuong, Q. H., La, V. P., Vuong, T. T., Ho, M. T., Nguyen, T. H. K., Nguyen, V. H., Pham, H. H., \& Ho, M. T. (2018). An open database of productivity in Vietnam's social sciences and humanities for public use. Scientific Data, 5, 180188. https://doi.org/10.1038/sdata.2018.188

Vuong, Q. H., Le, A. V., La, V. P., Hoang, P. H., \& Ho, M. T. (2020). Making social sciences more scientific: Literature review by structured data. MethodsX, 7, 100818. https://doi.org/10.1016/j.mex.2020.100818 